\title{
Fifth Congress of Industrial Cell Technology 2014
}

The highly specialized and informative Fifth Congress of Industrial Cell Technology took place in Luebeck, close to Hamburg, on 11-12 September 2014. It was organized by the Fraunhofer Institution for Marine Biotechnology (EMB), Luebeck and supported by the cluster agency Life Science Nord Management GmbH as well as the Luebeck Chamber of Industry and Commerce. The central aim of the congress was to promote the name-giving platform applications of industrial cell technologies, in other words, the development of complex cell culture systems, analyzing technologies, innovative instruments and materials, etc. This year's sessions were: smart cell culture, bioreactor systems and cell goods including 3D bioprinting. This article highlights selected presentations of the congress.

The talks of the first session discussed ways for smart cell culture.

Daniel Rapoport, PhD, EMB, Luebeck, Germany, shared his ideas and experiences about how to smarten cell culture. He said: "Statements like 'My cells are looking good' are not a scientific quality control," although using the microscope was the oldest technique to characterize and assess cell cultures. This brought Mr Rapoport to image-based cytometry: by using a toolbox of latest generation software algorithms, micrographs of cells can be generated and used to quantify cell cultures. Analysis of time series of micrographs allowed for migration analysis to investigate the response of immune or tumor cells to stimuli. His group developed a new technique to link the genealogic information to immune-cytochemical stainings of cells to analyze stem cell cultures.

Also, they developed a capsule-based approach to expansion of adherent cells, where they used the inner surface of porous hydrogels. Cells grow protected and encapsulated in millimeter-sized hydrogel spheres. At a specified density, the capsule could be dissolved and the released cells subsequently re-encapsulated again. The hydrogel matrix could be used to immobilize growth fac- tors and reporter molecules to improve monitoring of the cells.

Kristian Tryggvason, $\mathrm{PhD}$, BioLamina AB, Sundbyberg, Stockholm, Sweden, presented the Laminin (LN) proteins as the key to primary cell culture. This extracellular protein family has been known to be part of the extracellular matrix, and each cell type has its own set of LN. Sixteen different LN influence cell differentiation, adhesion, migration and phenotype stability. $\mathrm{He}$ described how LN521 was part of the inner cell mass of the blastocyst and coating it on plastic dishes would support long-term selfrenewal of embryonic and induced pluripotent stem cells in a xeno-free cell culture up 2 years.

The well-received talk of Andrea Traube, $\mathrm{PhD}$, Fraunhofer Institute for Production Technology and Automation IPA, Stuttgart, Germany, described the challenges of automated cell- and tissue culture. Although cell and tissue cultures were frequently used, the processes for generating cells and tissue models remained expensive, difficult and time-consuming. One major obstacle was the complex and personnel-intensive manufacturing protocol. Ms Traube described the first fully automated sterile manufacturing
Anja Rasch

Life Science Nord Management GmbH, Hamburg, Germany 
plant to produce 3D human epidermal skin equivalents ('Tissue Factory') using human keratinocyte cell suspension. The automated process included cell seeding, expansion and tissue differentiation on customized, robotics-compatible transwell inserts. The scientists achieved lot sizes of roughly 700 skin equivalents per week. Also, the epidermis equivalents produced in the Tissue Factory were shown to be comparable to manually cultured epidermis models. Ms Traube reminded the audience to consider the aim of automation of cell culturing, in other words, if throughput should be enhanced and cautioned: "It takes years to automate cell culture". She suggested saving time by parallel development of steps such as lab processes, device construction, process integration, adaption and validation. Despite the successful automation, Ms Traube said that it takes 6 months to set up a new protocol. For the future, she pointed to goals as to further minimize the automation system (current footprint $3 \times 3 \mathrm{~m}$ ) and to work on modularization as well.

The talks of the second session concentrated on bioreactors.

Gregor Liebsch from PreSens - Prescision Sension $\mathrm{GmbH}$ talked about integrated sensors for noninvasive monitoring that the company offers. Sensor technology can be applied for single-point measurements or $2 \mathrm{D}$ visualization of analyte distributions, either microinvasively or contactlessly. Tools for $2 \mathrm{D}$ mapping at $\mu \mathrm{m}$-scale in combination with microsensors also enabled for assessing biomaterials 3-dimensionally. Even multiplexing with 24-well plates, read-out of multiple sensors incorporated in bioreactors or custom systems could be performed for oxygen, $\mathrm{pH}$ and $\mathrm{CO}_{2}$. Core of this technology were fluorescent dyes, integrated in sensor foils or other sensor designs, which translate analyte concentration into light signals and can be read through the transparent vessel walls either with a digital handheld microscope or with an optical fiber.

Gerhard Greller, PhD, Sartorius Stedim Biotech $\mathrm{GmbH}$, Goettingen, Germany, told about the singleuse bioreactors. Scientists can scale up their cell cultures from 5 to $1000 \mathrm{l}$ reactor; in other words, Sartorius researchers had achieved a monoclonal antibody production and harvested a finaI IgG titer of $8 \mathrm{~g} / \mathrm{l}$ (7 kg IgG).

The third session presented examples of cell goods.

Markus Rimann, PhD, Zurich Academy for Applied Sciences (ZHAW), Zurich, Switzerland, presented his vision of organs on demand by using the bioprinting of 3D tissue models. He described bioprinting as a controlled layer-by-layer deposition of biological material to produce $3 \mathrm{D}$ organotypic in vitro models. Thus, the user would gain flexibility in the $3 \mathrm{D}$ shape and also spatial control of cells, matrix and bioactive molecules. In order to provide an all-in-one solution to print full-thickness skin equivalents, a printable and cyto-compatible matrix called BioInk (of undisclosed ingredients) was developed. In alternating layers of BioInk and cell suspension of dermal fibroblasts researchers built a dermis model. Onto the eight layers of the dermal cell construct, keratinocytes were seeded on top after 6 weeks and allowed to proliferate. As the polymerization of the BioInk was light-induced, a UVLED (365 nm) was integrated into the bioprinter. For in situ quality control a noninvasive optical coherence tomography system was used to distinguish between epidermis and dermis. The Swiss group adapted the bioprinter for sterile conditions. Mr Rimann said that currently a stirring system was installed to prevent sedimentation of cells. Around $80 \%$ of the cells would survive the printing process at room temperature. New projects included muscle-tendon cell models for drug development to investigate muscle contraction under electrical stimulation as well as formation of syncytia. Also in development were kidney and liver tissue models, expected to be suitable for drug toxicity tests.

Jonas Schödube, MSc, cytena GmbH, Freiburg, Germany, presented an inkjet-like single-cell printer as a tool for monoclonal cell line production and singlecell genomics. It could be used for the generation of clonal cell lines for biopharmaceuticals production, automated patch clamping of primary cells, genomic analysis of single cells, in other words, in tumor and prenatal diagnostics. Single-cell printing was based on optical imaging with a high-magnifying camera and automated image processing, allowing label-free detection, morphological analysis and classification before a one-cell-containing droplet is generated. For each cell, four images of the cell in the nozzle were stored as quality check and post analysis. A vacuum shutter was set up to remove droplets containing no or multiple cells. According to Schöndube, any water-based cell media could be used for cells with a size of 5-35 $\mu \mathrm{m}$ in a concentration range from $10^{3}$ to $10^{7}$ cells $/ \mathrm{ml}$. The achieved average cell viability was $80-90 \%$.

Lars Poulsen, $\mathrm{PhD}$, from ExpreS2ion Biotechnologies, Hørsholm, Denmark, praised the advantages of the Drosophila Schneider line 2 (S2) insect cell expression systems in recombinant subunit vaccine development: low-cost production, nonviral and nonlytic, high amount of protein and also allowing for a variety of upstream processing options. With Drosophila S2 cells researchers were able to produce hard-to-express proteins, hetero dimers and make glycosylation feasible. Poulsen stressed the cGMP compatibility of the system.

Kathrin Adlkofer, PhD, Bioenergy CellTec GmbH, Luebeck, described how to reach the goal of fast-healing 
wound-care therapy. Hydrophilic forms of biocompatible and resorbable poly-L-lactic polymers (Resomers) that degrade within 3-24 months are intended to be used as a matrix for autologous multipotent stem cells. The stem cells would be isolated from sweat-gland containing skin biopsies and have shown to form capillarylike structures in a cell model for dermal regeneration. The use of Resomers seeded with autologous stem cells might have the potential to promote wound healing, reduce scar tissue while keeping up the barrier function of the skin.

The keynote lecture reached out to philosophical topics and was held by Christoph Rehmann-Sutter, professor for theory and ethics of biosciences, University of Luebeck, Germany. The talk "Do we understand what we can build? Reprogramming and reimagination of cellular life" focused on a citation of Richard Feynman, Professor of Theoretical Physics at Caltech, Pasadena: "What I cannot create, I do not understand." Has one only understood when one can build it? What about the consequences of this insight? The knowledge that was necessary to synthesize cells would doubtlessly contribute to our understanding of life. Rehmann-Sutter said: "You still can philosophize about life, even when you can synthesize cells." Contrary to Feynman, J. Craig Venter, who had the first artificial genome synthesized of his Mycoplasma mycoides JCVI-syn 1.0, said in 2012: "What I cannot build, I cannot understand." Thereby, Venter would abstract cells to biomachines and digitalbiological converters. It would be tempting to naively assume all genomes could be as understandable as human language. That led Rehmann-Sutter to elaborate on the theory of understanding and what understanding meant. It was a surprising short step from understanding (something we do actively) to life and being alive. The latter was related to space and time, and beyond mere detection and description - a continuous-concrete event. What did we learn from biotechnology about life? Mr Rehmann-Sutter concluded that we did not understand life but we learned how to get around in living bodies. And when we would understand this epistemic relation of biotechnology to 'life' we would allow living things to be in the world in their own different ways.

For 2015, a symposium is scheduled in the new premises of the Fraunhofer EMB in Luebeck on 10-11 September 2015. Please visit the websites below for upcoming details:

- www.zelltechnik-kongress.de

- www.industrielle-zelltechnik.de

\section{Financial \& competing interests disclosure}

The author has no relevant affiliations or financial involvement with any organization or entity with a financial interest in or financial conflict with the subject matter or materials discussed in the manuscript. This includes employment, consultancies, honoraria, stock ownership or options, expert testimony, grants or patents received or pending, or royalties.

No writing assistance was utilized in the production of this manuscript. 